Review

\title{
Innovative Strategies and Challenges for the Prevention of Pathological Anxiety in Children and Adolescents
}

Ioannis Syros ${ }^{1,2,}{ }^{*}$, Aggeliki Karantzali ${ }^{3}$, Xenia Anastassiou-Hadjicharalambous ${ }^{4}$

1. Department of Child Psychiatry, School of Medicine, National and Kapodistrian University of Athens, "Aghia Sophia" Children's Hospital, Athens, Greece; E-Mail: jonsir9975@yahoo.com

2. Child and Adolescent Psychiatry Unit, "Sotiria" General Hospital, Athens, Greece

3. Society of Social Psychiatry, P.Sakellaropoulos, Athens, Greece; E-Mail: aggeliki.karantzali@yahoo.com

4. Psychology Program, University of Nicosia, 46 Makedonitissas Avenue, P.O.Box 24005, 1700 Nicosia, Cyprus; E Mail: hadjicharalambous.x@unic.ac.cy

* Correspondence: Syros loannis; E-Mail: jonsir9975@yahoo.com

Academic Editor: Tamas Kozicz

Special Issue: Novel Concepts of Mood and Anxiety in Children

OBM Neurobiology

2021, volume 5 , issue 3

doi:10.21926/obm.neurobiol.2103106
Received: December 16, 2020

Accepted: August 24, 2021

Published: September 13, 2021

\begin{abstract}
The effects of pathological anxiety on public health have led to the realization that anxious children might experience significant limitations in their lives. Evidence-based cognitivebehavioral (CB) interventions are now being implemented in schools at a group level to combat anxiety.Childhood emotional health should be considered a national and global priority for an effective strategy promoting the prevention of mental health. The aim of this study is the description of novel data on the effectiveness of preventive interventions for anxious children, as well as the study of selective parameters that appear to affect treatment outcome.Furthermore, a brief review is cited regarding two empirically supported interventions, which include the Friends and Pyramid Club program and the Super Skills for Life program. Schools are considered suitable places for all types of prevention programs; they
\end{abstract}

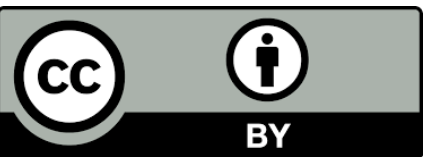

(c) 2021 by the author. This is an open access article distributed under the conditions of the Creative Commons by Attribution License, which permits unrestricted use, distribution, and reproduction in any medium or format, provided the original work is correctly cited. 
provide a familiar environment to a child, and by providing access to the prevention programs to many children, school programs could be the ideal way for anxious young individuals to practice and generalize their skills. Schools also offer an ideal space for collaboration between teachers and mental health professionals. Most programs have been effective in reducing anxiety, especially those based on the principles of $C B$, where the effects appear to be independent of the professional status of the leader (trained teacher or mental health professional). Friends and Pyramid Club interventions are well-established, multifaceted, and cost-effective programs, which include combinations of techniques (mostly behavioral activation, cognitive preparation, teaching social skills, and relaxation techniques). Super Skills for Life is a promising program, which also uses video feedback as a part of treatment. Prevention programs have been established as a vital part of the treatment options for specialists. Research related to childhood anxiety is increasing, while the perspectives for finding novel data seem promising for the upcoming years.In this context, several studies reveal the need for early, selective, intensive, persistent, multifaceted, and participatory interventions.

\section{Keywords}

Preventive interventions; anxiety disorders; pathological anxiety; children; adolescents; school-based programs; cognitive-behavioral therapy; evidence-based interventions

\section{Introduction}

Anxiety disorders (AD) occur in approximately $10 \%$ of children up to the age of 16 . They significantly reduce daily activities and often persist for a long time, thus increasing the risk of developing other psychiatric disorders in adolescence and young adulthood [1, 2].

In the Birth Dunedin Cohort study, $52-55 \%$ of young adults with clinical depression or pathological anxiety were diagnosed with emotional disorders before the age of 15 , while $75 \%$ were diagnosed with emotional disorders before the age of 18 [3]. Thus, pathological anxiety at a young age increases the risk of $A D$, clinical depression, substance abuse, and educational failure in early adulthood [4-7]. Additionally, pathological anxiety affects physical health as well as leads to financial and social costs $[8,9]$. Understanding the effects of pathological anxiety on public health has led to the realization that anxious children might experience significant limitations in their lives, even if they do not have a clinical disorder [10]. Therefore, the emotional health of children is necessary and should be considered a national and global priority, to develop a comprehensive and effective public health policy [11].

Effective psychological interventions at a group level, especially cognitive-behavioral therapy (CBT), are available for children with AD [12]. Additionally, studies suggest that CBT interventions at a group level are likely to be as effective as the interventions at an individual level in the prevention of emotional stress [13-16]. However, few children with AD are identified early and referred for treatment $[13,17]$. This finding highlighted the interest of the global community in conducting selective and persistent preventive interventions. 
Schools offer a "convenient" location to provide such programs [18, 19]. Systematic studies suggest that CBT prevention programs might be effective, although research is still inadequate methodologically, considering (a) field research protocols in everyday conditions are missing; (b) the effect sizes vary across studies; (c) randomized controlled trials (RCTs) conducted so far are few; (d) the influence of the head of the intervention (mental health professional vs. teacher) has been limited [2].

However, given that many of these programs do not target high-risk groups, it is argued that large effect sizes are not always expected. However, even small effect sizes across the population have therapeutic significance [20].

\section{Aim of the Study}

This study aimed to provide novel data on the effectiveness of empirically supported preventive interventions for children and adolescents with $A D$, as well as, to study various key parameters that affect the treatment outcome, such as the location of the program, the effect of the professional status of the intervention coordinator, and the value of supporting the content of the intervention with the theoretical framework of CBT therapy. We provided an overview of two empirically supported preventive interventions, i.e., the Friends and Pyramid Club program and the Super skills for Life program.

\section{Limited Access to Treatment Services}

Studies by Ford et al. [21] and Merikangas et al. [22] found that only $33 \%$ of children with AD, as well as $50 \%$ of children with depressive disorder, sought specialized psychological help during the study period from one to three years in UK and US populations. Additionally, compared to children with other mental health disorders, children with emotional disorders were less likely to receive help from these services. These data, combined with the limited availability of specialized treatment services so far, as well as the gradual shift of public views regarding health toward the early intervention of psychiatric disorders, show a growing interest in preventive interventions and the transition from clinical to community environments.

\section{Categories of Preventive Interventions}

Traditionally, Caplan [23] described three levels of prevention as primary, secondary, and tertiary. Primary prevention reduces the occurrence of psychopathology with interventions before the onset of a disorder. Secondary prevention seeks to reduce the prevalence of the pathology by intervening when the problems are identified but before the problems become clinically significant. Finally, tertiary prevention includes the treatment of existing disorders along with the prevention of relapse. However, the terminology of prevention provided by Gordon [24] is now considered the most widely accepted model. This model categorizes prevention interventions as Universal, Selective, and Indicated.

Universal preventive interventions are applied to entire populations, regardless of their emergency status. In some cases, universal preventive interventions can promote overall mental health or enhance resilience, while others are aimed at a specific disorder. 
On the contrary, selective preventive interventions are applied to those individuals who are at an increased risk of developing mental health disorders (e.g., parents of children with Autism, pregnant adolescent women, etc.).

Finally, indicative preventive interventions are applied to those individuals or groups who manifest mild symptoms but are at an extremely high risk of developing a full-blown mental disorder in the future (e.g., adolescents with disruptive behaviors and children with symptoms of anxiety).

\section{Advantages of Preventive Interventions in Schools}

As mentioned earlier, schools are considered suitable places for primary and secondary prevention programs $[18,19]$. They provide a familiar environment and access to the program to many children [25], which are the goals of the intervention. Additionally, schools offer affordable services so that specialized care can be provided to all [19,26, 27]. Finally, schools could be the ideal place for anxious young individuals to practice and generalize their skills, and for the identification of their psychopathology [18, 28, 29].

In summary, school programs targeting stress management and emotional problems have the following advantages:

a. Ease of finding candidate participants

b. Ease of implementing screening programs

c. Limited "leakage" of the participating students

d. Implementing programs for a wide range of psychosocial issues

e. Achieving maximum support from classmates

f. Providing perspectives for reducing psychosocial problems within the classroom, thereby promoting mental health and academic progress

Additionally, prevention programs in schools offer an ideal space for collaboration between teachers and mental health professionals. Although they might not be able to execute all aspects of the programs on their own, teachers and mental health professionals could act together, and their roles could complement those of one another. Teachers often believe that addressing problems of mental health is not an issue for the school to consider, and mental health specialists can resolve the issue. Additionally, teachers are often unaware of the usually easy-to-implement ways in which children with mental health disorders within the school can be supported. On the other hand, mental health professionals are often neither aware of the laws governing special education nor are they familiar with the "real-life" school routines and culture. Moreover, they are inexperienced in perceiving the obstacles in daily schoolwork, and sometimes they do not take into account the factors related to the practical implementation of such interventions in school [13, 18, 22, 30-32].

\section{Effectiveness of Prevention Programs in School}

Several reviews have been conducted so far, covering universal and selective/indicated approaches for children with emotional disorders, seeking to explore their effectiveness, as well as highlighting those factors that appear to affect their effectiveness in target populations. The reviews led to some common conclusions, such as:

a. Most universal and selective prevention programs were effective in reducing anxiety symptoms. 
b. Those programs that were based on a clear theoretical framework, especially CBT, were the most effective (e.g., teaching different skills such as relaxation, problem-solving, and awareness of the CBT model) [33].

c. The effects of CBT programs were marginally higher than those of non-CBT interventions, with the mean effect size, for the former, being 0.57 (moderate effect) [34]. However, there was a significant variation in the effect size of the results between the studies. The researchers stated that the large variation was due to the differences in the effectiveness of various factors, such as faithful adherence to the program, "bonding" with the "therapists", the organizational skills of the leader, and the level of participation and awareness of the target population in the intervention [33, 35]. Nevertheless, in a meta-analysis, the authors failed to identify an effect of the above variables [36].

However, there were methodological limitations in the evaluation studies of preventive interventions, such as (a) most interventions involved small sample sizes, (b) the use of nonstandardized instruments for measuring the outcome of the intervention, (c) a frequent lack of follow-up assessments, (d) the unresolved issue of why the effectiveness of the programs provided by teachers was superior to those provided by mental health professionals, and (e) the intervention protocols were missing field trials conducted for the daily and varied conditions in schools, which limited the generalizability of the effectiveness of these conditions $[2,18,31,37]$.

\section{Examining the Effect of the Leader of the Intervention: Mental Health Professional or School Teacher?}

Upon examining the effectiveness of school intervention programs, Neil et al. [33] found that program leaders were more likely to be mental health professionals, although, in $25 \%$ of the studies, the programs were coordinated by trained teachers, and these interventions also led to a significant reduction in stress. The authors argued that the effectiveness of the preventive interventions provided by the teachers, compared to those provided by mental health professionals, was unclear and was directly investigated in only a few studies where the FRIENDS program was implemented. But, in the reviews of Stallard et al. [2], Dadds \& Barrett [38], and Werner-Seidler et al. [39], it was found that the effectiveness of the intervention programs was similar between mental health professionals and trained teachers. However, in other applications of the FRIENDS program provided by school teachers, the researchers failed to find a positive result $[40,41]$, while some authors postulated that the effectiveness of health and school leaders differ depending upon the target of the program (e.g., anxiety vs. depression vs. externalized behaviors) and the essence of the outcome (emotional health vs. educational achievement) [42, 43]. In conclusion, it is still unclear whether the intervention programs led by teachers are as effective as those led by mental health staff.

\section{The Role of Cognitive Behavioral Therapy}

CBT provides a framework to help children understand the association between their thoughts, feelings, and behaviors. At a practical level, children identify, test, and reevaluate their non-helpful beliefs in more useful ways. CBT includes many behavioral techniques to facilitate behavioral changes, the most important of which are positive reinforcement of supportive behaviors, contingency management, self-assessment of negative thoughts, and gradual exposure [14, 44, 45]. The expected results of CBT relate to each of the following areas of the model: (a) in cognitive 
modification, resulting in the reduction of anxiety or negative thoughts, (b) in the improved emotional response, resulting in the reduction of anxiety and depressive symptoms, and (c) changes in behavior that result in improved self-efficacy as the child successfully learns to overcome and face challenges $[13,46,47]$.

\section{Presentation of Preventive Interventions for Childhood Anxiety with Well-Established Empirical Support}

There are many prevention programs for managing pathological anxiety in children and adolescents, and most are very similar $[36,48]$. Their core idea is that young individuals (and sometimes their parents, but also their teachers) can be taught specific skills to manage their emotional stress. Most programs include combinations of techniques. Below we will present three empirically supported and cost-effective interventions that are mostly based on the principles of cognitive-behavioral therapy.

\section{1 «FRIENDS» Program}

The "Friends" program is one of the best-evaluated programs, containing the most consistent indicators of effectiveness. This program has been endorsed by the World Health Organization [49] as an evidence-based program effective for managing anxiety in young individuals $[35,50,51]$. It was developed by the child psychologist, Professor Paula Barrett. Its development was based on the principles of the therapeutic program for anxiety disorders by Kendall, who called it "Coping Cat" $[52,53]$.

The "Friends" program is based on the principles of CBT, meaning that young participants develop skills to cope with the cognitive, emotional, and behavioral aspects of emotional stress. More analytically, children (a) develop emotional awareness and stress regulation skills, (b) replace their negative cognitive beliefs with more functional patterns of thinking, and (c) develop problemsolving skills to deal with distressing situations and events.

The description of the structure of the program is explained by the acronym:

$\mathbf{F}$ - Feeling worried?

R - Relax and feel good

I - Inner thoughts

E - Explore plans

$\mathbf{N}$ - Nice work so reward yourself

D - Do not forget to practice

S - Stay calm [33, 35, 49]

As mentioned above, the "Friends" program is based on significant evidence-based support. To demonstrate that, we provide the following data:

(a) In a randomized controlled trial conducted by Barret \& Turner [54], 489 children aged 10-12 years participated in the study. The self-reported measure of anxiety was significantly reduced in all the children who received the intervention in the teacher-led and the psychologist-led groups, compared to the anxiety levels in the children under the monitoring condition. Measurement of anxiety was based on validated scales (Spence Children's Anxiety Scale and Revised Child Manifest Anxiety Scale), and the analysis was based on mixed factorial ANOVA design. (b) The results were confirmed in two subsequent studies in which a sample of 594 children aged 10-13 years had 
participated, and the results remained consistent during the 12-month follow-up [55, 56]. (c) The "Friends" program also had a positive effect on the group that had a high score for stress; $85 \%$ of the individuals in that group were not diagnosed with $A D$ in the 12-month follow-up, compared to $31 \%$ of the individuals in the comparative group. (d) In a more recent study, which included 692 children, the "Friends» group showed a significant reduction in stress, which remained three years after the end of the program [57]. (e) In a study by Lock et al. [58], a comparison between children aged 9-10 years and those aged 14-16 years showed that both age groups benefited from the "Friends» program, while the younger group showed larger improvements in symptoms.

On the contrary, recent studies in Canada did not find additional benefits regarding the «Friends» program, either as a universal or a targeted intervention [40, 41].

The program has been adapted for four types of protocols based on the age of the participants: (a) Fun FRIENDS (4-7 years), (b) FRIENDS for life (8-11 years), (c) My FRIENDS Youth (12-15 years), and (d) Adult Resilience for Life (16 years and above).

This intervention has several structural advantages, compared to other intervention protocols. It is based on a clear theoretical model, which enhances the program design, as well as, is considered beneficial for the guidance, identification, development, and implementation of an effective intervention [33]. Furthermore, the sessions are sufficient, covering a wide range of evidence-based documented techniques and practices. There are up to 10 weekly sessions of 60-75 min, with two boost sessions of 60-75 min (after 1 and 3 months). Also, children are provided with a short homework session ("homework") in between sessions. Additionally, it is adapted for different age groups, providing the appropriate materials depending on the age, and includes enjoyable educational activities. It is based on a well-designed and comprehensive instruction manual with a detailed structure of each session $[57,59]$. The coordinators of the intervention receive clear and standardized training, and they might be supervised to ensure effective implementation of the program. The leader of the intervention meets the parents every week and provides them with contact sheets [30]. Two informative sessions are held by the leader of the intervention with the parents and the teachers for approximately 90-120 min to determine strategies to promote resilience at home and improve behavioral techniques for coping with anxiety in the classroom [2, $45,50]$. Meetings with parents are a very crucial part of the intervention. The meetings aim to inform parents to acknowledge and deal with their stress, as well as to train them in behavioral techniques (positive reinforcement, reward system, and distraction from challenging behavior). Additionally, meetings aim to educate parents in communication, mutual support within the family, and the acquisition of problem-solving skills. Finally, another essential aspect is the development of support networks between families and parents [14, 60].

One criticism of the FRIENDS program is that in the majority of the intervention programs, selfreport measures of anxiety were used to evaluate the outcome of the intervention. Children with $A D$ were reported to be more likely to provide socially desirable responses on self-report measures [61]. Additionally, it is unclear whether a significant change in the self-report symptom score reflects clinically important changes in everyday functioning [2]. Nevertheless, the degree of reliability of self-report measurements has been widely documented in the literature [62]. Moreover, clinicianrated diagnostic outcomes are difficult to perform, are costly, and time-consuming to administer [39]. Furthermore, it is questionable if CBT-based therapy is effective in the long run [13], and it is debatable whether this program, with appropriate cultural adaptations, regarding its content, could effectively help special target populations e.g. trauma - impacted refugees [41]. In a study by 
Skryabina et al. [43] with 1,362 participants, the authors failed to detect a favorable effect of the FRIENDS program, especially in academic performance, while in terms of the anxiety symptoms, the intervention was less effective when delivered by trained school staff, compared to the effectiveness of the intervention provided by mental health professionals.

\subsection{1 “Pyramid Club” Program}

It is a selective program developed in the early 1980 s by a social worker called Katrin Fitzherbert [63]. The main goal of the program is to improve self-esteem and social skills in children who are withdrawn, socially isolated, as well as, emotionally and mentally vulnerable [64].

It is one of the largest and well-established socio-emotional interventions in the United Kingdom, mainly in primary schools $[65,66]$, but also in secondary schools [67], targeting a wide range of children and adolescents aged 7-14 years. Pyramid Club is a 10-week group program that occurs once a week after school lessons for $90 \mathrm{~min}$.

According to the National Pyramid Trust [68], the goal of the program is to "build trust, improve the sense of well-being, and encourage friendship skills, allowing children to feel, perhaps for the first time in their lives, that they "really belong to ...".

The program consists of the following stages:

Stages 1 \& 2: Selection Process of the Participants. This is a two-step process. Initially, the "Strength and Difficulties Questionnaire» [69] is given to children at school to proceed to a screening process. Then, the children who are selected in the first step are discussed in a meeting at the school by those specialists (teachers, social workers, and school psychologists) who might be responsible for taking care of these children. Then, the children who are suitable to attend the pyramid group are selected.

Stage 3. In this stage, children who were selected earlier participate in pyramid groups ("clubs"). The clubs are structured and coordinated by volunteers who have been trained in Pyramid intervention methods, according to the relevant program manual. Specifically, volunteers are trained, through a three-day program, to organize activities (e.g., time cycle, club naming, etc.) and apply basic behavioral techniques (reward positive behavior, direct and selective reinforcement, function as positive role models).

Procedure. There are 10 weekly sessions held, aiming at developing social skills, building trust, and emotional expression. Each meeting includes the time cycle, group empowerment activities, and opportunities for children to practice their social skills in a safe and supportive environment. The first session starts by finding the name of the group and indicating four or five simple rules by the children, guided by the club coordinators. The aim is to enhance familiarity in the group by the children themselves.

Review at the End of the Intervention. A meeting is held among all the therapists and coordinators to discuss the progress of the entire intervention program and refer any child who might need further help.

Evaluation and Criticism of the Program. The strengths of this intervention are that it can be performed in small groups, emphasizes peer relationships, social well-being, emotional health, and 
emotional intelligence. These elements are considered critical components for the development of a child $[65,66,70,71]$. However, despite the program's acceptance and its support at a research level, some aspects could be criticized. To elaborate, clubs are guided by trained volunteers and not permanent staff; there are methodological limitations of the studies examining the effectiveness of the interventions; small sample size; the absence of a control group or waiting list; the partial evaluation of outcomes, based only on teachers' reports. Furthermore, the research related to the program is insufficient for the long-term assessment of therapeutic benefits. Moreover, it is not clear whether the improvement in socio-emotional health is associated with the improvement in other areas of functioning, for example, externalizing behaviors. Finally, the program is based on a vague theoretical background, along with the application of theoretical parts of CBT, components of positive psychology, and other approaches. [30, 59, 70].

\subsection{Super Skills for Life}

It is an intervention program for children between 6 and 18 years and is usually organized at schools to help young individuals cope with emotional difficulties such as anxiety disorders, depression, and comorbid symptoms [72]. Several studies have reported its immediate and longterm effects in the UK [73], Spain [74-76], and Mauritius [77]; additionally, promising preliminary results have been found in Greece [78]. Super Skills for Life is based on the principles of CBT and behavioral activation and uses video feedback and cognitive preparation as a part of the treatment. The effectiveness of video feedback with cognition-based methods to treat anxiety (especially social anxiety) has been widely investigated in adolescents and adults, and to a lesser extent, in the pediatric population.

There are two versions of the program: one is for children between 6 and 11 years, and the adolescent version is for children between 12 and 18 years. The program helps young people develop skills to a) improve self-confidence and emotional resilience, as well as improve social skills and abilities and b) cope with stressful situations, including changes in life, such as the transition from primary to secondary school. The adolescent version also covers health improvement techniques by promoting healthy eating, physical exercise, and good sleep hygiene $[59,72]$.

\subsubsection{Program Description}

Eight sessions are usually held once or twice a week. It can either be provided to a group of 6-8 children, or an individual, for $45 \mathrm{~min}$ per session. The sessions include role-playing games, homework, video analysis, group, and individual activities, and feedback. Each child receives a workbook to use in the sessions and at home, as well as, to practice their skills with the support of parents/caregivers.

An advantage of this program is that it can be provided by teachers and other non-mental health professionals after a short training session. Also, Super Skills for Life is considered a transdiagnostic intervention and is not targeted to specific disorders, for example, anxiety, depression, etc. Transdiagnostic interventions are especially important for prevention programs organized at schools, as they allow improvements in the psychological functioning of the participants [79, 80]. Furthermore, the use of video feedback with cognitive intervention provides extra benefits to the social performance of children, improving prosocial behaviors and communication skills, reducing discomfort in social situations, and increasing self-confidence [73, 81, 82]. Regarding the 
disadvantages of the intervention, the majority of the studies to date that focused on the effectiveness of the SSFL program did not include a control group to compare the findings. Trials with appropriate control groups are needed in future research to incorporate the results of untreated children, which will better elucidate the effectiveness of the program [83]. Additionally, this intervention does not include parents, although the effectiveness of parent support programs is also unclear, as several studies that incorporated parent support in the intervention programs were unable to evaluate their effects due to poor parental participation [34, 58, 84].

\section{Conclusion, Dilemmas, \& Future Challenges}

In the last two decades, there has been a tremendous expansion of the knowledge regarding the development and management of $A D$ in children and adolescents, although many key issues remain to be assessed. Nevertheless, we are currently at a point where children with anxiety are increasingly being acknowledged and can be well-evaluated.

The main advantages of the above-mentioned intervention programs could be summarized as follows: 1. All of them include various techniques and skills, which are considered transdiagnostic, meaning that they appear to have a favorable effect across various emotional disorders. For example, problem-solving skills are essential for young individuals with anxiety, but also those individuals with depression, withdrawal, procrastination, etc. 2 . The possibility for these programs to also be offered by trained teachers allows these programs to be conducted cost-effectively in schools. 3. Expanding the authors thinking which only refers to cases of social anxiety disorders in the relative study [85], group intervention has the advantage of providing a protective environment in which children have the opportunity to practice a variety of skills, although it is difficult to tailor to the specific needs of each child.

On the other hand, it is still questionable whether these programs are feasible in the school environment, and it remains debatable whether their effectiveness is ensured when the intervention leader is a trained teacher and not a mental health professional. Another criticism is that, in some cases, the evaluation of intervention studies was not based on data from multiple informants (children, parents, teachers), while, in most cases, researchers did not use diagnostic interviews, which would enhance the reliability of the results [45]. Finally, these group interventions might not be the most effective or efficient means of reducing anxiety in those children who are already experiencing clinically significant anxiety [54].

The prevention programs have been criticized by some researchers as they claim that the programs have not demonstrated consistent efficacy; however, they require money that could be invested in other mental health services. Additionally, the "detection process" creates moral issues, leaving aside people, who could be falsely negative in population screening, with "health certificates" [86]. Finally, as reported previously, it is doubtful if these intervention programs are effective, not only for emotional problems but also for the academic performance of the participants $[48,87]$.

However, prevention programs have gradually established their presence internationally as treatment options provided by specialists. Thus, research on prevention programs is increasing, and the perspectives are positive for the upcoming years. Early, selective, intensive, persistent, multifaceted, and participatory interventions are necessary. 
The following are the future challenges and suggestions regarding the research and implementation of prevention programs:

- A better understanding of the risk factors related to the development of stress, with the help of prospective studies, might contribute to specific prevention programs.

- Investigating the responsiveness and the possibilities of providing interventions for anxiety to special populations, such as refugees, people with coexisting chronic physical illness, etc.

- Sustainability of timely therapeutic gains; regarding this, Sandler [88] suggested that the effects of prevention programs should be determined by how well they change targeted outcomes over time, rather than through the determination of the immediate effects.

- The inclusion of booster and parent sessions also needs to be further promoted. For this, techniques must first be implemented to maximize parental involvement.

- The effectiveness of these intervention programs for high-anxiety children needs to be further investigated.

- Conducting detailed studies to identify which components of the whole intervention have comparatively the greatest therapeutic value; this finding is of great importance for the more targeted training of young health professionals.

- Promoting new methods of conducting prevention programs using technology, e.g., internet and remote programs (telehealth).

- Further research is needed to improve current methodological concerns regarding design and analysis, as well as participation and dropout rates. Additionally, independent studies need to be undertaken more often to ensure that researcher bias does not affect the results. Additional research on applied settings is also needed to determine if the intervention is best provided by trained teachers or mental health professionals and whether these programs are cost-effective and feasible in schools [45]. Furthermore, it is important to design interventions with control groups [33].

- It is essential to incorporate qualitative analysis while evaluating the overall effectiveness of these programs. This will provide additional data to complement the quantitative results obtained from the assessment of the views of children, teachers, and parents.

\section{Author Contributions}

Syros loannis conceived the original idea and wrote the manuscript. Karantzali Aggeliki and Anastassiou-Hadjicharalambous Xenia provided critical feedback and helped shape the manuscript.

\section{Funding}

This research did not receive any specific grant from funding agencies in the public, commercial, or not-for-profit sectors.

\section{Competing Interests}

The authors state that there are no conflicts of interest regarding the publication of this paper.

\section{References}


1. Voltas N, Hernández-Martínez C, Arija V, Canals J. The natural course of anxiety symptoms in early adolescence: Factors related to persistence. Anxiety Stress Coping. 2017; 30: 671-686.

2. Stallard P, Skryabina E, Taylor G, Anderson R, Ukoumunne OC, Daniels H, et al. A cluster randomised controlled trial comparing the effectiveness and cost-effectiveness of a schoolbased cognitive-behavioural therapy programme (FRIENDS) in the reduction of anxiety and improvement in mood in children aged 9/10 years. Southampton: NIHR Journals Library; 2015.

3. Kim-Cohen J, Caspi A, Moffitt TE, Harrington H, Milne BJ, Poulton R. Prior juvenile diagnoses in adults with mental disorder: Developmental follow-back of a prospective-longitudinal cohort. Arch Gen Psychiatry. 2003; 60: 709-717.

4. Donovan CL, Spence SH. Prevention of childhood anxiety disorders. Clin Psychol Rev. 2000; 20: 509-531.

5. Kessler RC, Keller MB, Wittchen HU. The epidemiology of generalized anxiety disorder. Psychiatr Clin North Am. 2001; 24: 19-39.

6. Birmaher B, Ryan ND, Williamson DE, Brent DA, Kaufman J, Dahl RE, et al. Childhood and adolescent depression: A review of the past 10 years. Part I. J Am Acad Child Adolesc Psychiatry. 1996; 35: 1427-1439.

7. Rao UM, Ryan ND, Birmaher B, Dahl RE, Williamson DE, Kaufman J, et al. Unipolar depression in adolescents: Clinical outcome in adulthood. J Am Acad Child Adolesc Psychiatry. 1995; 34: 566578.

8. Copeland WE, Angold A, Shanahan L, Costello EJ. Longitudinal patterns of anxiety from childhood to adulthood: The great smoky mountains study. J Am Acad Child Adolesc Psychiatry. 2014; 53: 21-33.

9. Fineberg NA, Haddad PM, Carpenter L, Gannon B, Sharpe R, Young AH, et al. The size, burden and cost of disorders of the brain in the UK. J Psychopharmacol. 2013; 27: 761-770.

10. Lyneham HJ, Rapee RM. Evaluation of therapist-supported parent-implemented CBT for anxiety disorders in rural children. Behav Res Ther. 2006; 44: 1287-1300.

11. Bittner A, Egger HL, Erkanli A, Jane Costello E, Foley DL, Angold A. What do childhood anxiety disorders predict? J Child Psychol Psychiatry. 2007; 48: 1174-1183.

12. Creswell $C$, Waite $P$, Hudson J. Practitioner review: Anxiety disorders in children and young people-assessment and treatment. J Child Psychol Psychiatry. 2020; 61: 628-643.

13. James AC, James G, Cowdrey FA, Soler A, Choke A. Cognitive behavioural therapy for anxiety disorders in children and adolescents. Cochrane Database Syst Rev. 2015; 2: CD004690.

14. Shortt AL, Barrett PM, Fox TL. Evaluating the FRIENDS program: A cognitive-behavioral group treatment for anxious children and their parents. J Clin Child Psychol. 2001; 30: 525-535.

15. Silverman WK, Pina AA, Viswesvaran C. Evidence-based psychosocial treatments for phobic and anxiety disorders in children and adolescents. J Clin Child Adolesc Psychol. 2008; 37: 105-130.

16. Barrett PM. Evaluation of cognitive-behavioral group treatments for childhood anxiety disorders. J Clin Child Psychol. 1998; 27: 459-468.

17. Reynolds S, Wilson C, Austin J, Hooper L. Effects of psychotherapy for anxiety in children and adolescents: A meta-analytic review. Clin Psychol Rev. 2012; 32: 251-262.

18. Herzig-Anderson K, Colognori D, Fox JK, Stewart CE, Warner CM. School-based anxiety treatments for children and adolescents. Child Adolesc Psychiatr Clin. 2012; 21: 655-668.

19. Masia-Warner C, Nangle DW, Hansen DJ. Bringing evidence-based child mental health services to the schools: General issues and specific populations. Educ Treat Children. 2006; 29: 165-172. 
20. Bayer J, Hiscock H, Scalzo K, Mathers M, McDonald M, Morris A, et al. Systematic review of preventive interventions for children's mental health: What would work in Australian contexts? Aust N Z J Psychiatry. 2009; 43: 695-710.

21. Ford $\mathrm{T}$, Hamilton $\mathrm{H}$, Meltzer $\mathrm{H}$, Goodman R. Predictors of service use for mental health problems among British schoolchildren. Child Adolesc Ment Health. 2008; 13: 32-40.

22. Merikangas KR, He JP, Brody D, Fisher PW, Bourdon K, Koretz DS. Prevalence and treatment of mental disorders among US children in the 2001-2004 NHANES. Pediatrics. 2010; 125: 75-81.

23. Caplan G. Principles of preventive psychiatry. New York: Basic; 1964.

24. Gordon Jr RS. An operational classification of disease prevention. Public Health Rep. 1983; 98 : 107-109.

25. Anglin TM. Mental health in schools: Programs of the federal government. In: Handbook of school mental health: Advancing practice and research. New York: Kluwer Academic/Plenum; 2003. pp.89-103.

26. Weist MD. Challenges and opportunities in expanded school mental health. Clin Psychol Rev. 1999; 19: 131-135.

27. Catron T, Weiss B. The Vanderbilt school-based counseling program: An interagency, primarycare model of mental health services. J Emot Behav Disord. 1994; 2: 247-253.

28. Evans SW, Langberg J, Williams J. Achieving generalization in school-based mental health. In: Handbook of school mental health: Advancing practice and research. New York: Kluwer Academic/Plenum; 2003. pp.335-348.

29. Evans SW. Mental health services in schools: Utilization, effectiveness, and consent. Clin Psychol Rev. 1999; 19: 165-178.

30. Skaloubakas C. Modification in school environments targeting to students anxiety management. Lecture in the postgraduate program "Anxiety Disorders in Children and Adolescents". Athens: University of Athens; 2016.

31. Adi Y, Killoran A, Janmohamed K, Stewart-Brown S. Systematic review of the effectiveness of interventions to promote mental wellbeing in children in primary education. Report 1: Universal approaches: Non-violence related outcomes. Coventry: University of Warwick; 2007. pp.149.

32. Department for Education and Skills. Excellence and enjoyment: Social and emotional aspects of learning. Guidance. London: Department of Education and Skills; 2005.

33. Neil AL, Christensen H. Efficacy and effectiveness of school-based prevention and early intervention programs for anxiety. Clin Psychol Rev. 2009; 29: 208-215.

34. Mifsud C, Rapee RM. Early intervention for childhood anxiety in a school setting: Outcomes for an economically disadvantaged population. J Am Acad Child Adolesc Psychiatry. 2005; 44: $996-$ 1004.

35. Fisak BJ, Richard D, Mann A. The prevention of child and adolescent anxiety: A meta-analytic review. Prev Sci. 2011; 12: 255-268.

36. Ahlen J, Lenhard F, Ghaderi A. Universal prevention for anxiety and depressive symptoms in children: A meta-analysis of randomized and cluster-randomized trials. J Prim Prev. 2015; 36: 387-403.

37. Shucksmith J, Summerbell C, Jones S, Whittaker V. Mental wellbeing of children in primary education (targeted/indicated activities) Database of Abstracts of Reviews of Effects (DARE): Quality-assessed reviews. England: University of York; 2007. 
38. Dadds MR, Barrett PM. Practitioner review: Psychological management of anxiety disorders in childhood. J Child Psychol Psychiatry. 2001; 42: 999-1011.

39. Werner-Seidler A, Perry Y, Calear AL, Newby JM, Christensen H. School-based depression and anxiety prevention programs for young people: A systematic review and meta-analysis. Clin Psychol Rev. 2017; 51: 30-47.

40. Miller LD, Laye-Gindhu A, Liu Y, March JS, Thordarson DS, Garland EJ. Evaluation of a preventive intervention for child anxiety in two randomized attention-control school trials. Behav Res Ther. 2011; 49: 315-323.

41. Miller LD, Laye-Gindhu A, Bennett JL, Liu Y, Gold S, March JS, et al. An effectiveness study of a culturally enriched school-based CBT anxiety prevention program. J Clin Child Adolesc Psychol. 2011; 40: 618-629.

42. Skryabina E, Taylor G, Stallard P. Effect of a universal anxiety prevention programme (FRIENDS) on children's academic performance: Results from a randomised controlled trial. J Child Psychol Psychiatry. 2016; 57: 1297-1307.

43. Calear AL, Christensen H. Systematic review of school-based prevention and early intervention programs for depression. J Adolesc. 2010; 33: 429-438.

44. David D, Cristea I, Hofmann SG. Why cognitive behavioral therapy is the current gold standard of psychotherapy. Front Psychiatry. 2018; 9: 4.

45. Higgins E, O'Sullivan S. "What Works": Systematic review of the "FRIENDS for Life" programme as a universal school-based intervention programme for the prevention of child and youth anxiety. Educ Psychol Pract. 2015; 31: 424-438.

46. Rapee RM, Schniering CA, Hudson JL. Anxiety disorders during childhood and adolescence: Origins and treatment. Annu Rev Clin Psychol. 2009; 5: 311-341.

47. Silverman WK, Kurtines WM. Anxiety and phobic disorders: A pragmatic approach. New York: Plenum Press, 1996.

48. Durlak JA, Weissberg RP, Dymnicki AB, Taylor RD, Schellinger KB. The impact of enhancing students' social and emotional learning: A meta-analysis of school-based universal interventions. Child Dev. 2011; 82: 405-432.

49. World Health Organization. Prevention of mental disorders: Effective interventions and policy options: Summary report/ a report of the World Health Organization Dept. of mental health and substance abuse \& the prevention research centre of the Universities of Nijmegen and Maastricht. Geneva: World Health Organization; 2004.

50. Stallard P, Simpson N, Anderson S, Goddard M. The FRIENDS emotional health prevention programme. Eur Child Adolesc Psychiatry. 2008; 17: 283-289.

51. Sulkowski ML, Joyce DK, Storch EA. Treating childhood anxiety in schools: Service delivery in a response to intervention paradigm. J Child Fam Stud. 2012; 21: 938-947.

52. Kendall P, Choudhury M, Hudson J, Webb A. The CAT project manual. Ardmore: Workbook Publishing; 2002.

53. Kendall PC. Treating anxiety disorders in children: Results of a randomized clinical trial. J Consult Clin Psychol. 1994; 62: 100-110.

54. Barrett $P$, Turner $C$. Prevention of anxiety symptoms in primary school children: Preliminary results from a universal school-based trial. Br J Clin Psychol. 2001; 40: 399-410.

55. Lowry-Webster HM, Barrett PM, Lock S. A universal prevention trial of anxiety symptomology during childhood: Results at 1-year follow-up. Behav Change. 2003; 20: 25-43. 
56. Lowry-Webster HM, Barrett PM, Dadds MR. A universal prevention trial of anxiety and depressive symptomatology in childhood: Preliminary data from an Australian study. Behav Change. 2001; 18: 36-50.

57. Barrett PM, Farrell $\amalg$, Ollendick TH, Dadds M. Long-term outcomes of an Australian universal prevention trial of anxiety and depression symptoms in children and youth: An evaluation of the friends program. J Clin Child Adolesc Psychol. 2006; 35: 403-411.

58. Lock S, Barrett PM. A longitudinal study of developmental differences in universal preventive intervention for child anxiety. Behav Change. 2003; 20: 183-199.

59. Syros I. Preventive interventions for children and adolescents anxiety disorders. In: Modern child and adolescent psychiatry: Mental health and psychopathology. Athens: Beta publication; 2020.

60. Silverman WK, Kurtines WM, Ginsburg GS, Weems CF, Lumpkin PW, Carmichael DH. Treating anxiety disorders in children with group cognitive-behavioral therapy: A randomized clinical trial. J Consult Clin Psychol. 1999; 67: 995-1003.

61. Dadds MR, Perrin S, Yule W. Social desirability and self-reported anxiety in children: An analysis of the RCMAS Lie Scale. J Abnorm Child Psychol. 1998; 26: 311-317.

62. Kendall PC, Flannery-Schroeder EC. Methodological issues in treatment research for anxiety disorders in youth. J Abnorm Child Psychol. 1998; 26: 27-38.

63. Pyramid Club. Leader training manual. London: Pyramid; 2007.

64. Munoz RF, Mrazek PJ, Haggerty RJ. Institute of medicine report on prevention of mental disorders: Summary and commentary. Am Psychol. 1996; 51: 1116-1122.

65. Cassidy T, McLaughlin M, Giles M. Socio-emotional health in school children: An emotionfocused intervention. Int J Sch Cogn Psychol. 2015; 2: 1-7.

66. McKenna ÁE, Cassidy T, Giles M. Prospective evaluation of the pyramid plus psychosocial intervention for shy withdrawn children: An assessment of efficacy in 7-to 8-year-old school children in Northern Ireland. Child Adolesc Ment Health. 2014; 19: 9-15.

67. Jayman $M$, Ohl M, Hughes B, Fox P. Improving socio-emotional health for pupils in early secondary education with Pyramid: A school-based, early intervention model. Br J Educ Psychol. 2019; 89: 111-130.

68. The National Pyramid Trust, Charity for education/training for children at risk, Charity Commision for England and Wales. 2005.

69. Goodman R. The strengths and difficulties questionnaire: A research note. J Child Psychol Psychiatry. 1997; 38: 581-586.

70. Cassidy T, McLaughlin M, Giles M. Group-based intervention to improve socioemotional health in vulnerable children. J Psychol Clin Psychiatry. 2014; 1: 00045.

71. OhI M, Fox $P$, Mitchell K. Strengthening socio-emotional competencies in a school setting: Data from the Pyramid project. Br J Educ Psychol. 2013; 83: 452-466.

72. Essau CA, Ollendick TH. The super skills for life programme. London: University of Roehampton; 2013.

73. Essau CA, Olaya B, Sasagawa S, Pithia J, Bray D, Ollendick TH. Integrating video-feedback and cognitive preparation, social skills training and behavioural activation in a cognitive behavioural therapy in the treatment of childhood anxiety. J Affect Disord. 2014; 167: 261-267. 
74. Fernández Martínez I, Morales Sabuco A, Espada Sánchez JP, Orgilés Amorós M, Essau CA. Effectiveness of the program super skills for life in reducing symptoms of anxiety and depression in young Spanish children. Psicothema. 2019; 31: 298-304.

75. Fernández-Martínez I, Orgilés M, Morales A, Espada JP, Essau CA. One-year follow-up effects of a cognitive behavior therapy-based transdiagnostic program for emotional problems in young children: A school-based cluster-randomized controlled trial. J Affec Disord. 2020; 262: 258-266.

76. Orgilés M, Fernández-Martínez I, Espada JP, Morales A. Spanish version of super skills for life: Short-and long-term impact of a transdiagnostic prevention protocol targeting childhood anxiety and depression. Anxiety Stress Coping. 2019; 32: 694-710.

77. Ramdhonee-Dowlot K, Balloo K, Essau CA. Effectiveness of the super skills for life programme in enhancing the emotional wellbeing of children and adolescents in residential care institutions in a low-and middle-income country: A randomised waitlist-controlled trial. J Affect Disord. 2021; 278: 327-338.

78. Tsourdini A, Syros I, Konsta M, Anastassiou-Hadjicharalambous X, Essau C. Preliminary implementation of the Greek adaptation of the psychoeducational intervention program "super skills for life" in the clinical pediatric population. Oral Presentation. Proceedings of the 8th Panhellenic Conference on Developmental and Behavioral Pediatrics. 2019; Athens, Greece.

79. Catalano RF, Fagan AA, Gavin LE, Greenberg MT, Irwin Jr CE, Ross DA, et al. Worldwide application of prevention science in adolescent health. Lancet. 2012; 379: 1653-1664.

80. Zins JE, Bloodworth MR, Weissberg RP, Walberg HJ. The scientific base linking social and emotional learning to school success. J Educ Psychol Consult. 2007; 17: 191-210.

81. Parr CJ, Cartwright-Hatton S. Social anxiety in adolescents: The effect of video feedback on anxiety and the self-evaluation of performance. Clin Psychol Psychother. 2009; 16: 46-54.

82. Ramos V, Piqueras JA, García-López L. Efficacy and efficiency of the use of video-feedback in the cognitive-behavioral treatment of young people with social anxiety disorder. Clin Salud. 2008; 19: 249-264.

83. Essau CA, Sasagawa S, Jones G, Fernandes B, Ollendick TH. Evaluating the real-world effectiveness of a cognitive behavior therapy-based transdiagnostic program for emotional problems in children in a regular school setting. J Affect Disord. 2019; 253: 357-365.

84. Barrett PM, Lock S, Farrell LJ. Developmental differences in universal preventive intervention for child anxiety. Clin Child Psychol Psychiatry. 2005; 10: 539-555.

85. Spence SH, Donovan C, Brechman-Toussaint M. The treatment of childhood social phobia: The effectiveness of a social skills training-based, cognitive-behavioural intervention, with and without parental involvement. J Child Psychol Psychiatry. 2000; 41: 713-726.

86. Harrington $\mathrm{R}$, Clark A. Prevention and early intervention for depression in adolescence and early adult life. Eur Arch Psychiatry Clin Neurosci. 1998; 248: 32-45.

87. Hoagwood KE, Serene Olin S, Kerker BD, Kratochwill TR, Crowe M, Saka N. Empirically based school interventions targeted at academic and mental health functioning. J Emot Behav Disord. 2007; 15: 66-92.

88. Sandler I. Progress in developing strategies and theory for the prevention and treatment of anxiety and depression. Prev Treat. 1999; 2: 9c. 


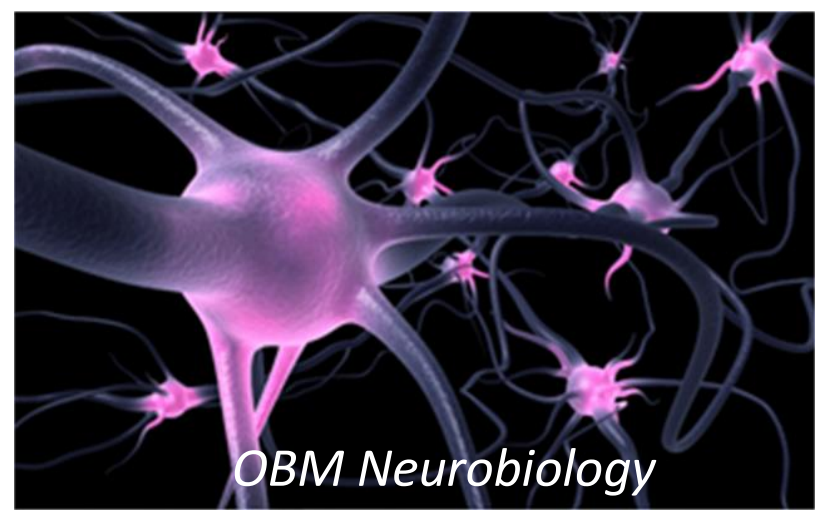

Enjoy OBM Neurobiology by:

1. Submitting a manuscript

2. Joining volunteer reviewer bank

3. Joining Editorial Board

4. Guest editing a special issue

For more details, please visit:

http://www.lidsen.com/journals/neurobiology 